\title{
The association between cannabis abuse and subsequent schizophrenia: a Swedish national co-relative control study
}

\author{
G. N. Giordano ${ }^{1}$, H. Ohlsson ${ }^{1}$, K. Sundquist ${ }^{1,2}$, J. Sundquist ${ }^{1,2}$ and K. S. Kendler ${ }^{3,4,5 *}$ \\ ${ }^{1}$ Center for Primary Health Care Research, Lund University, Malmö, Sweden \\ ${ }^{2}$ Stanford Prevention Research Center, Stanford University School of Medicine, Stanford, CA, USA \\ ${ }^{3}$ Virginia Institute for Psychiatric and Behavioral Genetics, Virginia Commonwealth University, Richmond, VA, USA \\ ${ }^{4}$ Department of Psychiatry, Virginia Commonwealth University, Richmond VA, USA \\ ${ }^{5}$ Department of Human and Molecular Genetics, Virginia Commonwealth University, Richmond, VA, USA
}

Background. Although cannabis abuse (CA) is known to be associated with schizophrenia, the causal nature of this association is unclear, with prodromal effects complicating its interpretation.

Method. From Swedish national registry databases, we used a co-relative case-control design with full-sibling, halfsibling and first-cousin comparisons, alongside a general Swedish population sample. Using ICD codes, 5456 individuals with an initial diagnosis of schizophrenia (2000-2010) were matched with five schizophrenia-free controls. We further identified first-cousin, half-sibling and full-sibling pairs discordant for CA and statistically extrapolated results for discordant monozygotic (MZ) twins.

Results. Within the general Swedish population, CA was strongly associated with later schizophrenia [odds ratio (OR) $10.44,95 \%$ confidence interval (CI) 8.99-12.11]. This association was substantially attenuated both by increasing temporal delays between CA exposure and schizophrenia diagnosis and by controlling for increasing degrees of familial confounding. Extrapolated discordant MZ pairs suggested that fully controlling for confounding familial factors reduced the association between CA and later schizophrenia to more modest levels (ORs of approximately 3.3 and 1.6 with 3- and 7-year temporal delays respectively). Opiate, sedative, cocaine/stimulant and hallucinogen abuse were also strongly associated with subsequent schizophrenia in the general population. After controlling for familial confounding, only cocaine/stimulant exposure remained associated.

Conclusions. CA has an appreciable causal impact on future risk for schizophrenia. However, population-based estimates of cannabis-schizophrenia co-morbidity substantially overestimate their causal association. Predictions of the cases of schizophrenia that might be prevented by reduced cannabis consumption based on population associations are therefore likely to be considerably overestimated.

Received 26 February 2014; Revised 23 May 2014; Accepted 1 June 2014; First published online 1 July 2014

Key words: Cannabis, causality, cocaine, co-relative design, schizophrenia.

\section{Introduction}

A long history of empirical evidence shows an association between cannabis use and symptoms of psychosis (for reviews, see D'Souza, 2007; Minozzi et al. 2010). Three main inferences may be drawn from this extensive body of research. First, cannabis intoxication may lead to transient psychotic episodes (Negrete et al. 1986; Thornicroft, 1990; Mathers \& Ghodse, 1992; Sewell et al. 2009). Second, cannabis use seems commonplace among individuals who are vulnerable to,

\footnotetext{
* Address for correspondence: K. S. Kendler, M.D., Virginia Institute for Psychiatric and Behavioral Genetics of VCU, Box 980126, Richmond, VA 23298-0126, USA.

(Email: kendler@vcu.edu)
}

or have pre-existing symptoms of, psychosis and/or schizophrenia (Linszen et al. 1994; Dixon, 1999; Degenhardt et al. 2003; Koskinen et al. 2010). Third, although there is empirical evidence to support the theory that cannabis use is a component cause of schizophrenia (Andreasson et al. 1987; Arseneault et al. 2002; van Os et al. 2002; Weiser et al. 2002; Zammit et al. 2002; Veling et al. 2008; McGrath et al. 2010), the 'etiologic' hypothesis remains controversial (Fergusson et al. 2006; Moore et al. 2007; McLaren et al. 2010; Sevy et al. 2010). In this study we focused on the third point and investigated the degree to which the association between cannabis use and schizophrenia is causal.

Evidence supporting causality, according to 'Hill's Postulates' (Hill \& Hill, 1991; Goodman \& Phillips, 2005), includes specificity of association (van Os et al. 
2002; Zammit et al. 2002) and a dose-related response (Arseneault et al. 2002; Veling et al. 2008; Di Forti et al. 2009; McGrath et al. 2010), with heavy cannabis users (cannabis abusers) having a sixfold increase in risk of future diagnosis of schizophrenia (Andreasson et al. 1987; Zammit et al. 2002).

Of all the suggested causal criteria, however, only temporality is labeled 'essential'; that is, exposure must occur prior to disease onset (Hill \& Hill, 1991; Goodman \& Phillips, 2005). Prodromal effects common to schizophrenia could make temporality difficult to establish; affected individuals may suffer from a variety of non-specific symptoms (anxiety, depression, concentration problems, etc.) that occur months to years prior to the manifestation of full schizophrenic illness (Klosterkotter et al. 2001; Cornblatt et al. 2003). During this time, individuals may use cannabis either as a result of the prodromal illness or in an attempt to treat their emerging symptoms. One review assessed current evidence of association between cannabis use and psychosis/schizophrenia alongside Hill's causal criteria and concluded that '[this observed] association may reflect a causal relationship' (McLaren et al. 2010). However, the same review recognized the need for future research '[to] control for important environmental and biological confounding factors'.

Given the consistent evidence for strong familial/ genetic contributions to both schizophrenia and cannabis use and misuse (Tsuang et al. 1996; Kendler \& Prescott, 1998; Kendler et al. 2000; Sullivan et al. 2003; Verweij et al. 2010), a substantial concern should be that some or all of the association between cannabis use and schizophrenia arises from familial confounding. However, to date, only one prospective study has attempted to control for such confounders by using a sibling-pair analysis (McGrath et al. 2010). Sibling pairs are a natural experiment; they each share $50 \%$ of their parents' genes, often share a similar prenatal environment and share similar environmental factors as they grow up (Lawlor \& Mishra, 2009). Investigating sibling pairs who are discordant for cannabis use, alongside a general population sample, permits an assessment of the degree to which observed associations between cannabis use and schizophrenia are confounded by familial (genetic and/or shared environmental) factors.

An extension of this methodology is the co-relative control design that includes other relatives, in particular half-sibling and first-cousin pair comparisons; half-siblings share on average $25 \%$ of their genes, and cousins $12.5 \%$. Shared environmental influences also decrease across the three co-relative groups, the presumption being that cousin pairs are the group likely to spend the least time together growing up. The use of a co-relative design may therefore provide a more convincing case as to the nature and extent of familial confounding, particularly if an expected gradient is observed, as familial confounding is increasingly controlled.

The aim of this study was to investigate the causal nature of the association between cannabis abuse (CA) and a future diagnosis of schizophrenia. We used longitudinal national data containing independent observations of CA prior to later hospital diagnosis of schizophrenia. We tested the etiologic hypothesis that CA is one direct cause of schizophrenia using a co-relative control design and then proceeded to consider the specificity of this association and temporal issues surrounding potential prodromal effects.

\section{Method}

Our study used linked data from multiple Swedish nationwide registries and healthcare data. Linking was achieved through the unique individual Swedish 10-digit personal ID number assigned at birth or immigration to all Swedish residents. Our databases used the following registers: the Total Population Register, containing annual data on family status; the Multi-Generation Register, providing information on family relations; the Swedish Hospital Discharge Register, containing all hospitalizations for Swedish inhabitants between 1964 and 2010; the Out-patient Care Register, containing information from all outpatient clinics between 2001 and 2010; the Swedish Crime Register, which included national complete data on all convictions from 1973 to 2011; and the Swedish Mortality Register, containing causes of death. We secured ethical approval for this study from the Regional Ethical Review Board of Lund University (No. 2008/409). Our methods for identifying CA and schizophrenia are given in the online Supplementary Appendix. Our definition of schizophrenia excluded diagnoses of simple schizophrenia, schizoaffective disorder and acute schizophrenia.

\section{Sample}

First, we identified all individuals in Sweden diagnosed with schizophrenia over the period 2000-2010. From these, we identified all individuals under 50 years of age at the time of their initial schizophrenia diagnosis $(n=5456)$. Each individual was then matched to five controls based on gender, age and country of birth, and without a diagnosis of schizophrenia from 1987 to 2010 . Our main exposure variable was CA. In both cases and controls, CA was defined only if the individual was registered as a cannabis abuser prior to the date of their first diagnosis of schizophrenia. We investigated the association between $\mathrm{CA}$ and 
individual schizophrenia risk in the general Swedish population using conditional logistic regression.

Second, we compared the results from the Swedish general population with results from a co-relative design. By means of the Swedish Multi-Generation Register, we identified all first-cousin, paternal and maternal half-sibling and full-sibling pairs who were born within 10 years of one another. We separated paternal and maternal half-siblings because, although they shared their degree of genetic resemblance, Swedish maternal half-siblings were much more likely to live together while growing up than paternal halfsiblings (Frisell et al. 2012b).

\section{Statistical analysis}

To adjust for an array of potentially confounding genetic and environmental factors, we used conditional logistic regression to examine all co-relatives pairs discordant for CA. In these analyses, only pairs discordant for schizophrenia are informative. If the individual with CA had a schizophrenia diagnosis, the CA registration had to be recorded prior to the schizophrenia diagnosis. Pairs in which one member had a registration of schizophrenia prior to year 2000 were excluded.

To consider potential prodromal effects, we also investigated the time between CA and schizophrenia diagnosis at 1-, 3-, 5- and 7-year intervals. Monozygotic (MZ) twin pairs discordant for CA exposure would be of particular interest in these analyses as they provide complete control for genetic and familial-environmental confounding. However, less than five $M Z$ twin pairs met our definition of discordance. Therefore, we estimated values for discordant $\mathrm{MZ}$ pairs based on the observed results from other relationships, that is by fitting a regression line to the $\beta$ coefficients from the five analyses (general population, cousins, paternal half-siblings, maternal half-siblings and siblings). We assumed values of additive genetic effects (A) from genetic expectations. The shared environmental estimates $(C)$ were calculated from the entire Swedish population born during the period 1970 to 1985 from a dataset for all possible relative pairs. Based on age difference, we calculated the percentage of possible time spent in the same household up to age 15. For example, a full sibling pair born 5 years apart that spent 10 years in the same household was given the value 1 whereas a pair born 5 years apart that spent 5 years in the same household was given the value 0.5 . The $A$ and $C$ mean estimates for each relative pair group were used for the extrapolation: population A:0, C:0; full cousins A:0.125, C:0; paternal half siblings: A:0.25, C:0.05; maternal half siblings A:0.25,
C:0.87; full siblings: A:0.5, C:0.95; and MZ twins: A:1, C:1.

We conducted two further sensitivity analyses to test the robustness of the results from our main analyses: (i) individuals had to have two separate registrations for both CA and later schizophrenia; and (ii) individuals with diagnoses of any drug psychoses and/or bipolar disorder were excluded from the schizophrenia cases (see online Appendix for ICD codes). We also tested the specificity of the association by investigating the association between CA and major affective disorder defined as bipolar or major depressive disorder (see online Appendix for ICD codes). Finally, we tested for substance specificity by investigating associations between abuse of other drug classes and later schizophrenia. All statistical analyses were performed using SAS version 9.3 (SAS Institute, 2008).

\section{Results}

From all schizophrenia cases derived from the Swedish general population, $10.28 \%$ were recorded as cannabis abusers prior to diagnosis, compared with $1.17 \%$ among controls. The mean number of days between registration for CA and subsequent diagnosis of schizophrenia was 2701 (s.D.=2355), that is approximately 7 years 3 months.

\section{General population sample}

As shown in Table 1, in the general population there was a large increased risk of diagnosis of schizophrenia if an individual had a prior registration of CA [odds ratio (OR) 10.44, 95\% confidence interval (CI) 8.99-12.11]. As the required time between CA and schizophrenia diagnosis was increased, this risk was attenuated but remained substantial and significant at 7 years (OR 4.24, 95\% CI 3.54-5.07).

\section{Co-relative control samples}

Table 1 also demonstrates the effect of the removal of familial confounding on the association between CA and a future schizophrenia diagnosis. As the degree of sharing of genetic and environmental factors increased (from first-cousin to full-sibling pairs), the association between CA and schizophrenia decreased (OR full-siblings 5.07, 95\% CI 4.17-6.16). As in the general population, the co-relative risk was attenuated as the required delay between CA and a schizophrenia diagnosis was increased. The risk for schizophrenia remained significant although modest at 7 years, even in full-sibling pairs (OR 1.98, 95\% CI 1.59-2.48). Table 1 also contains our extrapolated estimates for $\mathrm{MZ}$ twins discordant for CA. Controlling for all of the genetic and familial-environmental factors, we 
Table 1. Odds ratios (ORs) with $95 \%$ confidence intervals (CIs) describing the risk of hospital diagnosis of schizophrenia after prior registration for cannabis abuse (CA) in (i) a general population sample and (ii) a co-relative sample, allowing the time between exposure and disease to vary by $1,3,5$ and 7 years

\begin{tabular}{|c|c|c|c|c|c|}
\hline & At baseline & After 1year & After 3 years & After 5 years & After 7 years \\
\hline (i) General population sample & $\begin{array}{l}10.44(8.99-12.11) \\
(n=5456)\end{array}$ & $\begin{array}{l}9.19(7.89-10.70) \\
(n=5388)\end{array}$ & $\begin{array}{l}7.69(6.57-9.00) \\
(n=5306)\end{array}$ & $\begin{array}{l}5.95(5.04-7.02) \\
(n=5210)\end{array}$ & $\begin{array}{l}4.24(3.54-5.07) \\
(n=5119)\end{array}$ \\
\hline \multicolumn{6}{|l|}{ (ii) Co-relative sample } \\
\hline First-cousin pairs & $\begin{array}{l}9.40(8.12-10.87) \\
(n=2079)\end{array}$ & $\begin{array}{l}8.37(7.22-9.70) \\
(n=1836)\end{array}$ & $\begin{array}{l}7.19(6.19-8.35) \\
(n=1605)\end{array}$ & $\begin{array}{l}5.85(5.03-6.81) \\
(n=1343)\end{array}$ & $\begin{array}{l}4.05(3.46-4.73) \\
(n=989)\end{array}$ \\
\hline Paternal half-sibling pairs & $\begin{array}{l}9.15(6.39-13.11) \\
(n=342)\end{array}$ & $\begin{array}{l}8.42(5.87-12.09) \\
(n=328)\end{array}$ & $\begin{array}{l}7.82(5.44-11.23) \\
(n=298)\end{array}$ & $\begin{array}{l}6.12(4.24-8.84) \\
(n=239)\end{array}$ & $\begin{array}{l}4.18(2.86-6.11) \\
(n=173)\end{array}$ \\
\hline Maternal half-siblings pairs & $\begin{array}{l}6.00(4.20-8.58) \\
(n=245)\end{array}$ & $\begin{array}{l}5.85(4.04-8.46) \\
(n=226)\end{array}$ & $\begin{array}{l}4.70(3.23-6.84) \\
(n=188)\end{array}$ & $\begin{array}{l}3.42(2.32-5.05) \\
(n=146)\end{array}$ & $\begin{array}{l}2.58(1.72-3.85) \\
(n=118)\end{array}$ \\
\hline Full-sibling pairs & $\begin{array}{l}5.07(4.17-6.16) \\
(n=728)\end{array}$ & $\begin{array}{l}4.47(3.66-5.47) \\
(n=635)\end{array}$ & $\begin{array}{l}3.71(3.02-4.55) \\
(n=546)\end{array}$ & $\begin{array}{l}2.80(2.27-3.46) \\
(n=441)\end{array}$ & $\begin{array}{l}1.98(1.59-2.48) \\
(n=346)\end{array}$ \\
\hline MZ twins (extrapolated) & 3.92 & 3.38 & 3.31 & 2.63 & 1.67 \\
\hline
\end{tabular}

MZ, Monozygotic.

Table 2. Odds ratios (ORs) with 95\% confidence intervals (CIs) describing the risk of hospital diagnosis of schizophrenia after prior registration for cannabis abuse (CA) in (i) a general population sample and (ii) a co-relative sample

\begin{tabular}{llll}
\hline & $\begin{array}{l}\text { Two registrations for } \\
\text { schizophrenia and cannabis }\end{array}$ & $\begin{array}{l}\text { 'Purified' } \\
\text { schizophrenia }\end{array}$ & $\begin{array}{l}\text { Bipolar and major } \\
\text { depression }\end{array}$ \\
\hline (i) General population sample & $\begin{array}{l}13.57(10.49-17.55) \\
(n=3179)\end{array}$ & $\begin{array}{l}7.99(6.76-9.44) \\
(n=4398)\end{array}$ & $\begin{array}{l}2.98(2.75-3.17) \\
(n=42945)\end{array}$ \\
(ii) Co-relative sample & $9.88(8.16-11.96)$ & $9.88(8.16-11.96)$ & $2.48(2.35-2.61)$ \\
First-cousin pairs & $(n=1262)$ & $(n=1262)$ & $(n=6623)$ \\
& $12.29(7.50-20.16)$ & $6.89(4.60-10.32)$ & $1.73(1.51-1.98)$ \\
Paternal half-sibling pairs & $(n=231)$ & $(n=217)$ & $1.69(1.47-1.94)$ \\
& $5.91(3.76-9.28)$ & $5.56(3.63-8.51)$ & $(n=877)$ \\
Maternal half-siblings pairs & $(n=152)$ & $4.66(3.72-5.83)$ & $1.63(1.49-1.77)$ \\
& $4.37(3.45-5.51)$ & $(n=526)$ & $(n=2139)$ \\
Full-sibling pairs & $(n=446)$ & 3.53 & 0.80 \\
\hline
\end{tabular}

MZ, Monozygotic.

estimated ORs between CA and schizophrenia ranging from 3.92 with no time lag to 1.67 after 7 years.

\section{Sensitivity and specificity analyses}

As shown in Tables 2 and 3, after requiring two separate registrations of CA prior to two separate later diagnoses of schizophrenia, the observed association increased modestly in the general population but was similar or slightly attenuated in maternal half-sibling and full-sibling pairs and in our extrapolated discordant MZ twin pairs.

Having excluded all individuals with diagnoses of bipolar disorder (Lichtenstein et al. 2009) and/or any drug-induced psychosis prior to and after initial schizophrenia diagnosis, the association between CA and schizophrenia was slightly attenuated in the general population but little changed among close relatives and our estimated MZ pairs.

CA registration was significantly associated with a later diagnosis of major affective illness in the population (OR 2.98, 95\% CI 2.75-3.17). This association was decreased but remained significant in full-sibling pairs (OR 1.63, 95\% CI 1.49-1.77) but was fully attenuated in our extrapolated discordant MZ twin pairs.

Table 3 shows the observed associations between abuse of opiates, sedatives, cocaine/stimulants and hallucinogens and subsequent schizophrenia after 
Table 3. Odds ratios (ORs) with 95\% confidence intervals (CIs) describing the risk of hospital diagnosis of schizophrenia after prior registration for different types of drug abuse in (i) a general population sample and (ii) a co-relative sample (cannabis cases excluded)

\begin{tabular}{|c|c|c|c|c|}
\hline & Opiates & Sedatives & Cocaine/stimulants & Hallucinogens \\
\hline (i) General population sample & $\begin{array}{l}9.04(5.60-14.59) \\
(n=5456)\end{array}$ & $\begin{array}{l}12.74(9.52-17.04) \\
(n=5456)\end{array}$ & $\begin{array}{l}9.61(7.71-11.97) \\
(n=5456)\end{array}$ & $\begin{array}{l}26.67(15.36-46.29) \\
(n=5456)\end{array}$ \\
\hline \multicolumn{5}{|l|}{ (ii) Co-relative sample } \\
\hline First-cousin pairs & $\begin{array}{l}3.25(2.39-4.42) \\
(n=225)\end{array}$ & $\begin{array}{l}7.33(5.91-9.11) \\
(n=775)\end{array}$ & $\begin{array}{l}8.56(6.91-10.35) \\
(n=993)\end{array}$ & $\begin{array}{l}9.87(6.82-14.28) \\
(n=337)\end{array}$ \\
\hline Paternal half-sibling pairs & $\begin{array}{l}1.80(0.96-3.38) \\
(n=42)\end{array}$ & $\begin{array}{l}3.50(2.07-5.91) \\
(n=81)\end{array}$ & $\begin{array}{l}8.50(5.06-14.27) \\
(n=154)\end{array}$ & $\begin{array}{l}7.00(2.74-17.87) \\
(n=41)\end{array}$ \\
\hline Maternal half-siblings pairs & $\begin{array}{l}8.25(2.92-23.29) \\
(n=37)\end{array}$ & $\begin{array}{l}4.65(2.75-7.85) \\
(n=96)\end{array}$ & $\begin{array}{l}10.64(5.73-19.74) \\
(n=128)\end{array}$ & $\begin{array}{l}17.50(4.21-72.8) \\
(n=37)\end{array}$ \\
\hline Full-sibling pairs & $\begin{array}{l}1.49(1.01-2.19) \\
(n=107)\end{array}$ & $\begin{array}{l}3.34(2.51-4.43) \\
(n=269)\end{array}$ & $\begin{array}{l}4.36(3.21-5.93) \\
(n=268)\end{array}$ & $\begin{array}{l}5.73(3.31-9.92) \\
(n=101)\end{array}$ \\
\hline MZ twins (extrapolated) & $<1.00$ & $<1.00$ & 1.9 & $<1.00$ \\
\hline
\end{tabular}

MZ, Monozygotic.

excluding CA cases. In the general population, abuse of all four drug classes was strongly associated with schizophrenia, especially sedatives and hallucinogens. However, these associations were substantially attenuated in relative pairs. In our extrapolated discordant MZ twins, only cocaine/stimulant abuse remained associated with future schizophrenia risk.

\section{Discussion}

The aim of this study was to investigate the causal nature of the association between CA and the future diagnosis of schizophrenia using national-level data and a co-relative control design. As the risk for CA and schizophrenia runs strongly in families (Tsuang et al. 1996; Kendler \& Prescott, 1998; Kendler et al. 2000; Sullivan et al. 2003; Verweij et al. 2010), the results from a co-relative design could provide an important evaluation of the nature of the causal relationship between CA and schizophrenia.

Within the general Swedish population, CA was more strongly associated with later schizophrenia (OR 10.44) than has been observed in most prior studies (Arseneault et al. 2002; Zammit et al. 2002; Veling et al. 2008; Di Forti et al. 2009; McGrath et al. 2010). However, previous studies have suggested that the relationship between cannabis use is dose dependent and these prior studies nearly all examined only cannabis use. Because our sample of cannabis abusers had high enough levels of cannabis use to experience adverse legal or medical consequences, the association with schizophrenia would probably be stronger than that observed in studies only assessing cannabis use. Of note, after allowing for the same 5-year prodromal period as Zammit et al. (2002), our study produced similar results within the Swedish general population
(OR 5.95) to those identified as cannabis abusers within Swedish conscripts (OR 6.70).

Allowing 7 years from initial CA registration to later diagnosis, the risk for schizophrenia in discordant fullsibling pairs remained almost twofold. Our extrapolated MZ estimates suggest that, if familial factors were fully controlled for, the positive association between CA and later schizophrenia remained (OR 1.67). The results of this study therefore lend support to the etiologic hypothesis, that CA is one direct cause of later schizophrenia (Andreasson et al. 1987; Arseneault et al. 2002; van Os et al. 2002; Weiser et al. 2002; Zammit et al. 2002; Veling et al. 2008; McGrath et al. 2010). The strength of the association from our full-sibling analysis is similar to that derived from the meta-analysis performed by Arseneault et al. (2004); however, it is important to note that the latter's outcome definition is broader than the one used in this study, including schizophreniform and other psychotic symptoms.

Our results also suggest that a large part of the CA-schizophrenia association observed in the general population is not causal and results from confounding due to shared familial factors. The pattern of ORs observed in Table 1 gives some insight into the nature of these familial factors. The monotonic decline in ORs with increasing genetic resemblance in co-relative pairs suggests that shared genetic risk factors contribute substantially to the CA-schizophrenia association. However, the consistently lower ORs seen in maternal versus paternal half-siblings suggest that familial environmental factors also influence the co-occurrence of CA and schizophrenia.

The results of our sensitivity analyses strengthen our main findings. The 'double registration' analysis increased the rigor of both $\mathrm{CA}$ and schizophrenia 
diagnoses and found broadly similar patterns of association. The 'purified' schizophrenia analysis decreased the general population association (OR 7.99) but produced little overall change in the co-relative sample (compared with Table 1). Our first specificity analysis of the association between CA and major affective illness showed, at the population level, a significant association, albeit less robust than that seen between CA and schizophrenia. However, when controlling for familial confounding, especially with our extrapolated MZ twin pairs, the CA-affective illness association disappeared. These results suggest that, in contrast to the CA-schizophrenia relationship, the CA-affective illness association is not likely to be causal but instead results from confounding due to shared familial risk factors.

Past research investigating polydrug use implicated cannabis as the substance most likely to be associated with a later diagnosis of schizophrenia (Arseneault et al. 2002; van Os et al. 2002; Zammit et al. 2002). Our 'other substance' specificity tests (Table 3) show that, in the general population, associations between abuse of opiates, sedatives, cocaine/stimulants and hallucinogens and risk for subsequent schizophrenia were very similar to those seen for cannabis. These results are surprising, given the general lack of evidence of psychotogenic potential for opiates and sedatives (Brown \& Stoudemire, 1998; Dalmau et al. 1999). However, an examination of our co-relative results, including our extrapolated MZ twins, suggests that the associations for opiates, sedatives and hallucinogens were probably non-causal, arising instead from familial confounding. By contrast, our analyses suggest that exposure to cocaine and non-cocaine stimulants (at the level sufficient to be registered for abuse in Sweden) has a modest causal impact on future schizophrenia risk. This result is consistent with prior studies showing that stimulant abusers had an increased risk for psychosis (Mitchell \& Vierkant, 1991; Chen et al. 2003) and a subsequent diagnosis of schizophrenia (Post, 1975; Callaghan et al. 2012).

Overall, our results support the hypothesis that CA of sufficient severity to be detected in Swedish registries has an appreciable causal impact on future risk for schizophrenia. However, our findings also suggest that raw estimates of the cannabis-schizophrenia association substantially overestimate their causal association. Examination of Table 1 suggests that fully correcting for familial confounding reduces the CA-schizophrenia association by approximately twothirds. Although these results reflect those found in the meta-analysis performed by Arseneault et al. (2004), the latter's broader outcome definition may have led to an overestimation of cases of schizophrenia that might be prevented by reduced cannabis consumption, based on population associations.

\section{Strengths and limitations}

A major strength of our study is its annual sampling of a national population from 1987 to 2010, enabling us to perform the first co-relative study of CA and later schizophrenia. Our medical data are almost complete for exposure and outcome diagnoses. Nevertheless, there are several limitations of our study that should be noted.

First, by using registry data, we relied on a hospitalbased diagnosis of schizophrenia. Many consider the gold standard to be a 'research-based' diagnosis. However, an evaluation of Swedish diagnostic procedures concluded that schizophrenic psychoses in Swedish register data had 'high positive predictive power to a standard research DSM-IV diagnosis' (Ekholm et al. 2005).

Second, we identified CA from medical and legal records, using ICD and conviction codes to capture prevalence within our study population. Although this method has the important advantage of not requiring accurate respondent recall and self-reporting, the risk for misclassification bias remains. Furthermore, we have assumed that those admitted to hospital or convicted for cannabis use represented a subsample of heavy cannabis users, which are labeled 'cannabis abusers' in this study (i.e. it is likely that there were many more people who used/abused cannabis than those who were registered as CA). Therefore, some risk remains that $\mathrm{CA}$ identification in the current sample may be contaminated by evidence of prodromal schizophrenia. Because our subjects experienced adverse medical or legal consequences of their cannabis use, our results are not directly comparable to studies that examine cannabis use or even heavy cannabis use.

Third, it has been shown that in sibling and twin pair comparisons, estimates could be more biased by non-shared confounders than in unpaired (general population) estimates (Frisell et al. 2012a). However, it is unlikely that the effects of non-shared bias would have the same influence across the four relative groups of our co-relative control design. Furthermore, that our results showed a decreasing gradient of association as familial factors increased provides a convincing case as to the nature and extent of familial confounding of the association between CA and schizophrenia. We further extrapolated our $\mathrm{MZ}$ twin estimates from the regression lines of our co-relative models. Although we weighted these for genetic (A) and shared environmental (C) factors, the extrapolated results will not be as robust as those derived from our actual sample and should be interpreted accordingly. In addition, ORs greater than one in our sibling or simulated MZ pairs need not imply a causal link between CA and schizophrenia. Such results could arise, 
totally or in part, due to environmental experiences not shared with the sibling that increase risk for both CA and schizophrenia.

Fourth, only information pertaining to first registration for CA and first admission for schizophrenia were available in the registries. Therefore, we do not in fact know when the abuse or illness started. We explored this question by examining various temporal delays between CA and first schizophrenia admission to rule out the possibility that CA arose during the psychotic prodrome. As the delay becomes longer, the number of false-positive associations (excluded cases where CA arose as a result of the prodrome and did not causally contribute to schizophrenia) probably declines but the number of false negatives (excluded cases where CA did causally contribute to schizophrenia) also probably increases. We cannot determine what delay provides the most accurate picture of the causal association.

\section{Conclusions}

In the Swedish population investigated, CA was strongly associated with subsequent schizophrenia. However, controlling for familial confounding and prodromal effects substantially reduced later schizophrenia risk, intimating that a large proportion of the observed association was non-casual. However, as shown in our full-sibling comparisons, the results of this study provide empirical evidence lending further support to the hypothesis that CA is one component cause of schizophrenia. Current and future policies should consider this, along with the other reported deleterious health outcomes associated with cannabis use, when debating the legal status of cannabis.

\section{Supplementary material}

For supplementary material accompanying this paper visit http://dx.doi.org/10.1017/S0033291714001524.

\section{Acknowledgments}

Funding for this study was provided by National Institute on Drug Abuse (NIDA) Grant R01 DA030005, the Swedish Research Council to K.S. (K2012-70X-15428-08-3), the Swedish Research Council for Health, Working Life and Welfare (in Swedish: Forte; Reg. no: 2013-1836) to K.S., the Swedish Research Council to J.S. (2012-2378) and Access to Learning Fund (ALF) funding from Region Skåne awarded to J.S. and K.S.

\section{Declaration of Interest}

None.

\section{References}

Andreasson S, Engstrom A, Allebeck P, Rydberg U (1987). Cannabis and schizophrenia. A longitudinal study of Swedish conscripts. Lancet 2, 1483-1486.

Arseneault L, Cannon M, Poulton R, Murray R, Caspi A, Moffitt TE (2002). Cannabis use in adolescence and risk for adult psychosis: longitudinal prospective study. British Medical Journal 325, 1212-1213.

Arseneault L, Cannon M, Witton J, Murray RM (2004). Causal association between cannabis and psychosis: examination of the evidence. British Journal of Psychiatry 184, 110-117.

Brown TM, Stoudemire A (1998). Psychiatric Side Effects of Prescription and Over-the-Counter Medications: Recognition and Management. American Psychiatric Publishing: Chicago, IL.

Callaghan RC, Cunningham JK, Allebeck P, Arenovich T, Sajeev G, Remington G, Boileau I, Kish SJ (2012). Methamphetamine use and schizophrenia: a population-based cohort study in California. American Journal of Psychiatry 169, 389-396.

Chen CK, Lin SK, Sham PC, Ball D, Loh EW, Hsiao CC, Chiang YL, Ree SC, Lee CH, Murray RM (2003). Pre-morbid characteristics and co-morbidity of methamphetamine users with and without psychosis. Psychological Medicine 33, 1407-1414.

Cornblatt BA, Lencz T, Smith CW, Correll CU, Auther AM, Nakayama E (2003). The schizophrenia prodrome revisited: a neurodevelopmental perspective. Schizophrenia Bulletin 29, 633-651.

Dalmau A, Bergman B, Brismar B (1999). Psychotic disorders among inpatients with abuse of cannabis, amphetamine and opiates. Do dopaminergic stimulants facilitate psychiatric illness? European Psychiatry 14, 366-371.

Degenhardt L, Hall W, Lynskey M (2003). Testing hypotheses about the relationship between cannabis use and psychosis. Drug and Alcohol Dependence 71, 37-48.

Di Forti M, Morgan C, Dazzan P, Pariante C, Mondelli V, Marques TR, Handley R, Luzi S, Russo M, Paparelli A, Butt A, Stilo SA, Wiffen B, Powell J, Murray RM (2009). High-potency cannabis and the risk of psychosis. British Journal of Psychiatry 195, 488-491.

Dixon L (1999). Dual diagnosis of substance abuse in schizophrenia: prevalence and impact on outcomes. Schizophrenia Research 35, S93-S100.

D'Souza DC (2007). Cannabinoids and psychosis. International Review of Neurobiology 78, 289-326.

Ekholm B, Ekholm A, Adolfsson R, Vares M, Osby U, Sedvall GC, Jonsson EG (2005). Evaluation of diagnostic procedures in Swedish patients with schizophrenia and related psychoses. Nordic Journal of Psychiatry 59, 457-464.

Fergusson DM, Poulton R, Smith PF, Boden JM (2006). Cannabis and psychosis. British Medical Journal 332, 172-175.

Frisell T, Oberg S, Kuja-Halkola R, Sjolander A (2012a). Sibling comparison designs: bias from non-shared confounders and measurement error. Epidemiology 23, 713-720. 
Frisell T, Pawitan Y, Langstrom N, Lichtenstein P (2012b). Heritability, assortative mating and gender differences in violent crime: results from a total population sample using twin, adoption, and sibling models. Behavior Genetics 42, 3-18.

Goodman K, Phillips C (2005). Hill's criteria of causation. In Encyclopedia of Statistics in Behavioral Science (ed. B. S. Everitt and D. C. Howell), pp. 818-820. John Wiley \& Sons: Chichester, UK.

Hill AB, Hill ID (1991). Principles of Medical Statistics. Edward Arnold: London.

Kendler KS, Karkowski LM, Neale MC, Prescott CA (2000). Illicit psychoactive substance use, heavy use, abuse, and dependence in a US population-based sample of male twins. Archives of General Psychiatry 57, 261-269.

Kendler KS, Prescott CA (1998). Cannabis use, abuse, and dependence in a population-based sample of female twins. American Journal of Psychiatry 155, 1016-1022.

Klosterkotter J, Hellmich M, Steinmeyer EM, Schultze-Lutter F (2001). Diagnosing schizophrenia in the initial prodromal phase. Archives of General Psychiatry 58, 158-164.

Koskinen J, Lohonen J, Koponen H, Isohanni M, Miettunen J (2010). Rate of cannabis use disorders in clinical samples of patients with schizophrenia: a meta-analysis. Schizophrenia Bulletin 36, 1115-1130.

Lawlor D, Mishra G (2009). Family Matters: Designing, Analysing and Understanding Family-based Studies in Life-course Epidemiology. Oxford University Press: Oxford, UK.

Lichtenstein P, Yip BH, Bjork C, Pawitan Y, Cannon TD, Sullivan PF, Hultman CM (2009). Common genetic determinants of schizophrenia and bipolar disorder in Swedish families: a population-based study. Lancet 373, 234-239.

Linszen DH, Dingemans PM, Lenior ME (1994). Cannabis abuse and the course of recent-onset schizophrenic disorders. Archives of General Psychiatry 51, 273-279.

Mathers DC, Ghodse AH (1992). Cannabis and psychotic illness. British Journal of Psychiatry 161, 648-653.

McGrath J, Welham J, Scott J, Varghese D, Degenhardt L, Hayatbakhsh MR, Alati R, Williams GM, Bor W, Najman JM (2010). Association between cannabis use and psychosis-related outcomes using sibling pair analysis in a cohort of young adults. Archives of General Psychiatry 67, 440-447.

McLaren JA, Silins E, Hutchinson D, Mattick RP, Hall W (2010). Assessing evidence for a causal link between cannabis and psychosis: a review of cohort studies. International Journal of Drug Policy 21, 10-19.

Minozzi S, Davoli M, Bargagli AM, Amato L, Vecchi S, Perucci CA (2010). An overview of systematic reviews on cannabis and psychosis: discussing apparently conflicting results. Drug and Alcohol Review 29, 304-317.
Mitchell J, Vierkant AD (1991). Delusions and hallucinations of cocaine abusers and paranoid schizophrenics: a comparative study. Journal of Psychology 125, 301-310.

Moore THM, Zammit S, Lingford-Hughes A, Barnes TRE, Jones PB, Burke M, Lewis G (2007). Cannabis use and risk of psychotic or affective mental health outcomes: a systematic review. Lancet 370, 319-328.

Negrete JC, Knapp WP, Douglas DE, Smith WB (1986). Cannabis affects the severity of schizophrenic symptoms: results of a clinical survey. Psychological Medicine 16, 515-520.

Post RM (1975). Cocaine psychoses: a continuum model. American Journal of Psychiatry 132, 225-231.

SAS Institute (2008). SAS Online Documentation. Version 9.3. SAS Institute Inc.: Cary, NC.

Sevy S, Robinson DG, Napolitano B, Patel RC, Gunduz-Bruce H, Miller R, McCormack J, Lorell BS, Kane J (2010). Are cannabis use disorders associated with an earlier age at onset of psychosis? A study in first episode schizophrenia. Schizophrenia Research 120, 101-107.

Sewell RA, Ranganathan M, D'Souza DC (2009). Cannabinoids and psychosis. International Review of Psychiatry 21, 152-162.

Sullivan PF, Kendler KS, Neale MC (2003). Schizophrenia as a complex trait: evidence from a meta-analysis of twin studies. Archives of General Psychiatry 60, 1187-1192.

Thornicroft G (1990). Cannabis and psychosis. Is there epidemiological evidence for an association? British Journal of Psychiatry 157, 25-33.

Tsuang MT, Lyons MJ, Eisen SA, Goldberg J, True W, Meyer JM, Eaves LJ (1996). Genetic influences on abuse of illicit drugs: a study of 3,297 twin pairs. American Journal of Medical Genetics 67, 473-477.

van Os J, Bak M, Hanssen M, Bijl RV, de Graaf R, Verdoux H (2002). Cannabis use and psychosis: a longitudinal population-based study. American Journal of Epidemiology 156, 319-327.

Veling W, Mackenbach JP, van Os J, Hoek HW (2008). Cannabis use and genetic predisposition for schizophrenia: a case-control study. Psychological Medicine 38, 1251-1256.

Verweij KJ, Zietsch BP, Lynskey MT, Medland SE, Neale MC, Martin NG, Boomsma DI, Vink JM (2010). Genetic and environmental influences on canabis initiation and problematic use: a meta-analysis of twin studies. Addiction 105, 417-430.

Weiser M, Knobler HY, Noy S, Kaplan Z (2002). Clinical characteristics of adolescents later hospitalized for schizophrenia. American Journal of Medical Genetics 114, 949-955.

Zammit S, Allebeck P, Andreasson S, Lundberg I, Lewis G (2002). Self reported cannabis use as a risk factor for schizophrenia in Swedish conscripts of 1969: historical cohort study. British Medical Journal 325, 1199-1201. 